\title{
Influence of Alcohol Use on Neural Response to Go/No-Go Task in College Drinkers
}

\author{
Aral Ahmadi', Godfrey D Pearlson*,1,2,3, Shashwath A Meda', Alecia Dager',2, Marc N Potenza ${ }^{2,3,4}$, \\ Rivkah Rosen ', Carol S Austad ${ }^{5}$, Sarah A Raskin ${ }^{6}$, Carolyn R Fallahi ${ }^{5}$, Howard Tennen ${ }^{7}$, Rebecca M Wood ${ }^{5}$ \\ and Michael C Stevens ${ }^{1,2}$ \\ 'Olin Neuropsychiatry Research Center, Hartford Hospital/Institute of Living, Hartford, CT, USA; ${ }^{2}$ Department of Psychiatry, Yale University, \\ New Haven, CT, USA; ${ }^{3}$ Department of Neurobiology, Yale University, New Haven, CT, USA; ${ }^{4}$ Child Study Center, Yale University, New Haven, CT, \\ USA; ${ }^{5}$ Department of Psychology, Central Connecticut State University, New Britain, CT, USA; ${ }^{6}$ Department of Psychology, Trinity College, \\ Hartford, CT, USA; 'Department of Community Medicine and Health Care, University of Connecticut Health Center, Farmington, CT, USA
}

Impaired inhibition of prepotent motor response may represent an important risk factor for alcoholism. Alcohol use may also increase impulsive behavior, including impaired response inhibition. Little is known about the brain function underlying response inhibition among college-age drinkers based on their drinking patterns, despite college-age drinkers demonstrating high rates of alcohol-use disorders. Our major objective was to compare behavior and associated brain activity measured with $\mathrm{M} R \mathrm{RI}$ during a response-inhibition task in matched heavy- and light-alcohol-drinking college students. Participants were light $(N=36)$ and heavy $(N=56)$ drinkers, aged I8-20 years. We characterized blood oxygen level-dependent (BOLD) responses, while participants performed an fMRI Go/No-Go task to quantify inhibitory behavior and brain activity. Behaviorally, group performance differences were observed for Go correct-hit and No-Go falsealarm reaction times with increased reaction times in heavy compared with light drinkers. During fMRI No-Go correct rejections, light drinkers exhibited greater BOLD response than did heavy drinkers in left supplementary motor area (SMA), bilateral parietal lobule, right hippocampus, bilateral middle frontal gyrus, left superior temporal gyrus, and cingulate gyrus/anterior cingulate cortex (Brodmann area 24). Group differences in Go/No-Go-related regional activations correlated with alcohol- and impulsivity-related measures. These findings suggest that heavy alcohol drinkers may have dysfunction in brain regions underlying attention and response inhibition, leading to diminished abilities to suppress prepotent responding. The extent to which these tendencies relate to impulsive decision-making and behaviors in real-life settings and may guide intervention development warrants additional investigation.

Neuropsychopharmacology (2013) 38, 2197-2208; doi:10.1038/npp.2013.119; published online 12 June 2013

Keywords: fMRI; alcohol; adolescence; Go/No-Go task; college students

\section{INTRODUCTION}

Understanding psychological and neural processes, both leading to and consequent on alcohol-use disorders (AUDs), is important for both public health and addiction neuroscience. Adolescence is a high-risk period for initiating alcohol use and developing problem drinking. Most drinkers begin alcohol use in their teens and the greatest rates of alcohol abuse and dependence are between 18 and 25 years (SAMHSA, 2011; Dager et al, 2013). College students drink more and have higher rates of AUDs than same-aged non-college peers (Borsari, 2007).

\footnotetext{
*Correspondence: Professor GD Pearlson, Olin Neuropsychiatry Research Center, Hartford Hospital/Institute of Living, 200 Retreat Avenue, Hartford, CT 06106, USA, Tel: +1 8605457757 , Fax: + I 860545 7797, E-mail: godfrey.pearlson@yale.edu Received 8 January 2013; revised 19 April 2013; accepted 20 April 2013; accepted article preview online 14 May 2013
}

Impulsivity is an important component of drug and alcohol addiction susceptibility (Dalley et al, 2011; Lejuez et al, 2005, 2010; Oberlin and Grahame, 2009; Petry, 2001), including susceptibility based on familial risk (Knop, 1985; Petry et al, 2002; Saunders et al, 2008; Sher, 1991; Cloninger, 1987; Ernst et al, 2006). Impulsivity is a multifaceted construct (Meda et al, 2009) widely implicated in the development and maintenance of addictive behaviors (Verdejo-Garcia et al, 2008). Distinct aspects of impulsive behavior have been shown to influence alcohol-use initiation, escalation, and dependence differently (Courtney et al, 2012). Neural mechanisms of impulsivity point to dorsal anterior cingulate involvement with risk taking and correlation with hazardous drinking behavior (Claus and Hutchison, 2012).

Impulsive tendencies can be assessed using self-report questionnaires such as the Barratt Impulsiveness Scale (BIS-11) (Patton et al, 1995); such measures detect elevated impulsivity in regular users of multiple substances and those with familial histories of alcoholism (Verdejo-Garcia 
et al, 2008). Two broad impulsivity subtypes are choice impulsivity (involving rapid temporal discounting) and rapid response impulsivity (involving inhibition of prepotent responses); both have important implications for AUDs (Potenza and De Wit, 2010). A genetic susceptibility (predisposition) to addiction associated with increased impulsivity may manifest behaviorally as impaired response inhibition that may result from abnormal inhibitory control (Goldstein and Volkow, 2002; Kreek et al, 2005). Deficient inhibitory control is reported in multiple neuropsychiatric conditions, including alcohol/substance-use disorders (Bauer, 2001; Kaufman et al, 2003; Kouri et al, 1996) and externalizing disorders frequently linked to addiction risk, including antisocial/conduct-related disorders and attention-deficit hyperactivity disorder (Brandeis et al, 2002; Frank et al, 1998; Pliszka et al, 2000; Rubia et al, 1998). It is possible that pre-existing deficiencies in impulsive control might lead to AUD. However, the question of impulsivityAUD causality remains a circular one, the answer to which is still largely undetermined.

A major response-inhibition task is the Go/No-Go, where a prepotent bias towards fast responding to 'Go' stimuli increases the difficulty of withholding responses to 'No-Go' stimuli. Groups characterized by clinically relevant impulsivity (eg, AUDs) show diminished inhibition of responses to No-Go stimuli and thus make more errors of commission (ie, false-alarm responses on No-Go trials, when responses should be withheld/suppressed) (Kaufman et al, 2003; Chamberlain and Sahakian, 2007; Fillmore and Rush, 2002). Prior reports also show binge/heavy drinkers to perform significantly slower compared with their lighterdrinking counterparts, in response inhibition and directed attention tasks (Marczinski et al, 2007; Cox et al, 1999). Measures associated with effortful suppression are regarded as correlates of response inhibition (Pandey et al, 2012), and slow relative to fast stoppers may have weaker inhibition processes and abnormal error processing (Chamberlain and Sahakian, 2007; Lansbergen et al, 2007a, b). Functional MRI versions of the Go/No-Go task consistently show activation of multiregion neural networks (Rubia et al, 2001, Stevens et al, 2007, 2009, Posner and DiGirolamo, 1998; Smith and Jonides, 1999; Barkley, 1997; Weisbrod et al, 2000; Kaiser et al, 2003). Various regions within these networks are likely associated with particular aspects of the task, for example, anterior cingulate cortex (ACC) with choice and error/ conflict monitoring, and dorsolateral prefrontal cortex (DLPFC) with higher-order cognitive control over behavior. Moreover, broad networks likely form subcircuits, where groups of network nodes that are engaged by overall task performance might more directly correspond to specific cognitive operations. For example, Stevens et al (2007) showed that correct stops (No-Go's) engaged a subcircuit comprising left lateral prefrontal cortex, left postcentral gyrus/inferior parietal lobule, striatum, motor/premotor areas, and left cerebellum. Error commission engaged an another subcircuit that was not integrated with activity in regions engaged for higher-order cognitive control over behavior (eg, DLPFC). A third medial/dorsolateral prefrontal-parietal circuit responded to all No-Go stimuli, but with greater BOLD activity to errors.

Acute alcohol consumption influences both error processing and associated fMRI responses underlying No-Go false alarms (Anderson et al, 2011). However, abused substances may cause enduring changes in brain function that persist after cessation of use, leading to a 'hijacking' of brain reward, motivation, memory, and control circuits (Volkow et al, 1992) and damage to brain regions within neural networks. Specific brain regions (eg, DLPFC and hippocampus) and white matter tracts may be particularly vulnerable to such effects and considerably more so in developing brains of adolescents and young adults (Spear, 2000; Tomlinson et al, 2004). In the context of AUD, recent evidence in an adolescent sample supports the hypothesis that diminished neural activity during response inhibition predicts future involvement with problematic behaviors such as alcohol and substance abuse (Norman et al, 2011). Neural correlates of AUD during response inhibitions tasks show higher measures of AUD associated with lower functioning in regions including insula, inferior frontal gyrus, inferior parietal lobule, and anterior cingulate, and also greater engagement of motor response circuits preinhibition (Claus et al, 2013).

Behavioral measures such as number of blackouts may be indicators of rapid alcohol consumption (ie, gulping rather than drinking more steadily and slowly), which may in part reflect poor impulse control (Goodwin, 1995; Perry et al, 2006). Previous work has also demonstrated increased frontocerebellar response to a Go/No-Go task in substancenaive youth who later experience alcohol-related blackouts, suggesting pre-existing abnormalities in inhibitory processing that underlie blackout propensity (Wetherill et al, 2013). Twin studies indicate that drinking measures such as maximum drinks are highly heritable (Slutske et al, 1999) and are emphasized as an important endophenotype in large-scale alcohol dependence studies such as the Collaborative Study on the Genetics of Alcoholism (COGA) (Saccone et al, 2000).

On the basis of impulsivity-related findings and prior reports of altered fMRI and behavioral responses on Go/No-Go paradigms in impulsive or alcoholic individuals, we hypothesized that collegiate heavy drinkers would show: (a) higher scores on impulsivity-related measures; (b) impaired Go/No-Go response inhibition gauged by RTs and/or error rates (eg, slower RTs on Go correct-hit trials); and (c) diminished fMRI BOLD response patterns involving ACC, frontal cortical, motor cortical, striatal, and hippocampal responses during response inhibition. In line with the blunted response hypothesis, we also predicted that Go/No-Go neural responses would correlate negatively with out-of-magnet alcohol- and impulsivityrelated measures.

\section{MATERIALS AND METHODS}

\section{Subjects}

Participants were 92 young adults aged 18-20 years, recruited during their freshman year as part of an ongoing longitudinal study of alcohol and neurocognitive function (the Brain and Alcohol Research in College Students (BARCS) study; Dager, 2013). All subjects reported any alcohol consumption as well as any related consequences (eg, arrests, memory blackouts, missed classes) monthly, for the preceding month, in considerable detail on a secure 
Table I Demographic and Alcohol Use Characteristics of Study Participants

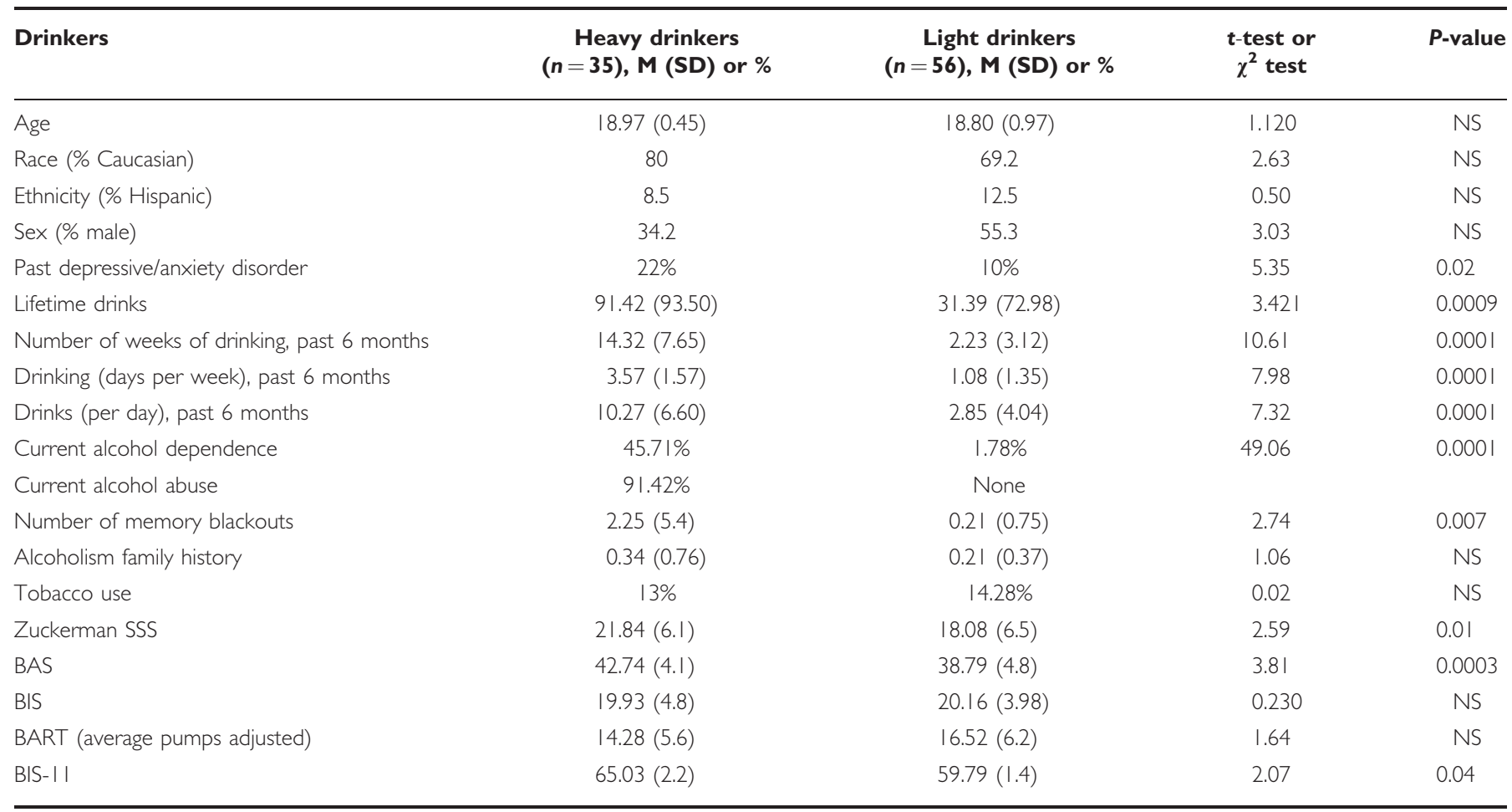

Abbreviations: BART, Balloon Analog Risk Task; BAS, Behavioral Activation System; BIS, Behavioral Inhibition System; BIS-I I, Barratt Impulsiveness Scale; M, mean; NS, nonsignficant; SD, standard deviation; Zuckerman SSS, Sensation Seeking Scale.

Groups did not differ on measures of age, sex, race, or family history of alcoholism, but they differed on drinking behavior in the past 30 days or 6 months and some impulsivity-related and anxiety measures.

website for 24 months. A subset of BARCS subjects participated in a neuroimaging study. All participants provided written informed consent, approved by the institutional review boards at Yale University, Hartford Hospital, Trinity College, and Central Connecticut State University. Exclusion criteria included current/past psychotic or bipolar disorders based on the Mini-International Neuropsychiatric Interview (MINI) (Sheehan et al, 1998), lifetime head injury that resulted in unconsciousness that lasted more than $5 \mathrm{~min}$, positive urine pregnancy test in women, or toxicology screen for abused substances. Eligible participants were evaluated for family-history-of-alcoholism genograms and personal drinking histories. Heavy drinking was defined using a combination of AUD diagnosis and quantity/frequency of current alcohol consumption (Cahalan et al, 1969; Squeglia et al, 2009; Dager et al, 2013). Participants were considered light drinkers if they: (1) did not meet current or past criteria for an AUD; and (2) drank during fewer than half of the weeks during the preceding 6 months. Participants were considered heavy drinkers if they either (1) met the criteria for current AUD or (2) drank more than half of the weeks in the preceding 6 months and reported that they typically binge-drank when drinking $(\geqslant 4$ drinks per occasion for females or $\geqslant 5$ drinks per occasion for males; eg, Dager et al, 2013; Courtney and Polich, 2009; Schweinsburg et al, 2010). The final sample of 56 light and 36 heavy drinkers did not differ on sex, ethnicity, race, family history (FH) of alcoholism and tobacco use (see Table 1 for demographics).

\section{Data Collection and Measures}

Detailed alcohol use history (memory blackouts, maximum drinks, frequency of drinking) was obtained using the alcohol-use module of the SCID (First et al, 2002). Current and past DSM-IV axis I diagnoses, including substance-use disorders, were ascertained using the MINI and information on daily tobacco use obtained using a Health Screening Questionnaire. At the time of scanning, participants were free of alcohol and illicit substances, as verified by breathalyzer and urine toxicology. Smokers could use tobacco up to $30 \mathrm{~min}$ before scan sessions.

Self-reported impulsivity was assessed with the BIS-11 (Patton et al, 1995), Behavioral Inhibition System/Behavioral Activation System (BIS/BAS) scale (Carver and White, 1994) and Zuckerman Sensation Seeking Scale (Zuckerman et al, 1979), with psychometric properties described in the referenced articles. The BIS-11 has been found to factor into three subscales assessing motor, non-planning, and attentional impulsivity (Patton et al, 1995). The Balloon Analog Risk Task (BART; Lejuez et al, 2002) was used to assess risk-taking, with higher values on the adjusted average of the total number of pumps on unexploded balloons indicative of greater risk-taking propensity (Bornovalova et al, 2005; Lejuez et al, 2002; Wallsten et al, 2005). 


\section{Go/No-Go Task}

We used an event-related fMRI task design described previously (Anderson et al, 2011; Kiehl et al, 2000a, b; Stevens et al, 2007). Participants were instructed to respond by pressing a button with their right index finger as accurately and quickly as possible to Go stimuli (' $\mathrm{X}$ ', $85 \%$ probability) and to withhold a response to No-Go stimuli ('K', $15 \%$ probability). Go and No-Go stimuli were presented for $50 \mathrm{~ms}$ with an interstimulus interval of 750 , 1750 , or $2750 \mathrm{~ms}$. The presentation of Go and No-Go stimuli was pseudorandomized with intervals of $10-15 \mathrm{~s}$ between No-Go stimuli. Trials were presented in 2 runs of 246 trials each lasting $7 \mathrm{~min} 21 \mathrm{~s}$, accounting for a total of 492 total trials per subject. A break of approximately $1 \mathrm{~min}$ was provided between runs. Given the high frequency of Go stimuli, the estimated response function was saturated and it was not possible to extract a meaningful result to Go correct-hit stimuli. Thus, imaging results are presented only for No-Go correct rejections $>$ baseline (successful inhibitions) and No-Go false alarms $>$ baseline (unsuccessful inhibitions) only.

Before scanning, participants completed 10 practice trials to ensure that instructions were understood. RT and accuracy were equally emphasized in task instructions. Participants were not given precise instructions about what number of errors was typical, nor provided with feedback during the task on the number of errors or speed of responses, information that could be used to adjust behavior. Participants were encouraged to perform at this level during both sessions to ensure that within-subject performance differences were meaningful.

\section{Data Analysis}

Go/No-Go behavioral analysis. The main behavioraldependent variable was response inhibition, indexed by RT and error proportion. RT data (in-scanner behavior) were presented separately for Go correct-hit and No-Go false-alarm trials. Although many Go/No-Go studies examine only Go correct-hit RTs, we also examined NoGo false-alarm RTs, as No-Go false alarms are believed to be primarily due to the failure to inhibit an impulsive response. RTs for Go correct hits and No-Go false alarms were analyzed for two groups (heavy and light drinkers). Error proportion (ie, false alarms) for No-Go trials was calculated as the number of errors on No-Go trials divided by the total number of No-Go trials. We analyzed both between-group differences and correlations against fMRI response patterns for impulsivity-related data including BIS/BAS (Carver and White, 1994), Zuckerman Sensation Seeking Scale (Zuckerman et al, 1979), BIS-11 (Patton et al, 1995), and BART (Lejuez et al, 2002). We also analyzed correlations between self-reported drinking behavior scores including maximum drinks (the largest number of alcoholic drinks consumed in $24 \mathrm{~h}$ (both for the past 6 months and lifetime based on Wechsler et al, 1999)), and alcohol-related memory blackouts (derived from a brief Young-Adult Alcohol Consequences Questionnaire (Kahler et al, 2005)).

Image acquisition. All images were acquired with a Siemens (Erlangen, Germany) Allegra $3 \mathrm{~T}$ high-performance head-dedicated system with $40 \mathrm{mT} / \mathrm{m}$ gradients and a standard quadrature head coil at the Olin Neuropsychiatry Research Center. Functional images were acquired axially using an echo planar image (EPI) gradient-echo pulse sequence covering the whole brain $(\mathrm{TR} / \mathrm{TE}=1500 / 28 \mathrm{~ms}$, flip angle $=65^{\circ}, \quad F O V=24 \mathrm{~cm} \times 24 \mathrm{~cm}, 64 \times 64$ matrix, $3.4 \mathrm{~mm} \times 3.4 \mathrm{~mm}$ in-plane resolution, $5 \mathrm{~mm}$ effective slice thickness, 30 slices).

Functional image analysis. Functional images from Go/No-Go were preprocessed using SPM5 (Department of Imaging Neuroscience, UK; http://www.fil.ion.ucl.ac.uk/ $\mathrm{spm} / \mathrm{software} / \mathrm{spm} 5 /$ ). Differences in EPI slice acquisition timing were corrected using the central slice as a reference. Motion was corrected using INRIAlign (Freire et al 2002), and images were then spatially normalized into Montreal Neurological Institute (MNI) space. Normalized EPIs were then smoothed with a 9-mm FWHM Gaussian kernel. Realignment parameters were examined for excessive motion, and participants with movement $>4.5 \mathrm{~mm}$ or $>3^{\circ}$ were not included in analyses. Before first-level analysis, events for each participant were categorized as correct hits to Go stimuli, correct rejects to No-Go stimuli, and false alarms to No-Go stimuli. For first-level analyses, a canonical hemodynamic response function and its temporal derivative (Josephs et al, 1997) were fitted to the onset of these three stimulus types for each session separately. Realignment parameters were included in the model as covariates of no interest.

Whole-brain group analysis. Group random-effects analyses were conducted to examine differences in activity between groups in each contrast, by entering contrast images of correct rejects $>$ baseline (successful inhibition) and false alarms $>$ baseline (unsuccessful inhibition) for each group into a post hoc t-test model. This explored between-group activity differences across the whole brain, using the unmodeled data as an implicit baseline. Post hoc $t$-test results were thresholded using a combination of voxel-wise $p=0.025$, false discovery rate-corrected (FDR-corrected) and a cluster extent of $k=25$ voxels.

For better interpretation of functional differences in each contrast, we also conducted a within-group results for each condition using a one-sample $t$-test, thresholded using the same parameters as above.

Region of interest analysis. We explored if impulsivity measures (BAS, BIS, BIS-11, Zuckerman, and BART) and alcohol-related measures (maximum alcohol drinks, number of blackouts) correlated with primary clusters that activated as main effects of group differences in each contrast using SPSS statistics IBM version 19. We examined relationships between functional changes and the abovedetailed measures by correlating the mean weighted $\beta$-estimates extracted from primary clusters that showed group difference in each contrast using MARSBAR (http:// marsbar.sourceforge.net/). 


\section{RESULTS}

\section{Demographics}

Demographic and alcohol-use characteristics of study participants were compared among groups using $\chi^{2}$ and $t$-tests (see Table 1). There were no between-group differences on sex, race, age, tobacco use, or alcoholism FH. Approximately $15 \%$ of participants (10\% of light and $22 \%$ of heavy drinkers) met the criteria for a past depressive or anxiety disorder. Compared with light drinkers, heavy drinkers reported greater levels of lifetime alcohol consumption, more memory blackouts, greater number of drinks per week, greater amounts of drinking during the past 6 months, and higher BAS, BIS-11, and sensation-seeking scores. BIS and BART scores did not differ between groups.

\section{Behavioral Results}

Behavioral results are summarized in Table 2.

Go correct hits. The groups differed on Go correct-hit RTs $(t(89)=-2.95, p=0.004$; Figure 1a). The RT slowing was marginally larger in heavy drinkers than in light drinkers. RTs for Go-correct hits also correlated positively with maximum alcohol-consumption scores (the largest number of alcoholic drinks that subjects consumed in $24 \mathrm{~h}$ (both lifetime and past 6-month measures) $\left(r_{\text {lifetime }}(89)=0.242\right.$, $p<0.02 ; r_{6}$ months $\left.(89)=0.245, p<0.02\right)$ ).

No-Go false alarms. Although groups did not differ in error proportions, they differed on No-Go false-alarm RTs $(t(89)=-2.94, p=0.004$; Figure $1 \mathrm{~b})$ with increased RT in heavy compared with light drinkers. RTs for No-Go false-alarm trials correlated with the number of reported memory blackouts $(r(89)=0.267, p<0.01)$ and maximum alcohol consumption (lifetime and past-6-month measures $)\left(r_{\text {lifetime }}(89)=0.288, p<0.006 ; r_{6}\right.$ months $(89)=0.323$, $p<0.003)$.

\section{Functional Results}

No-Go correct rejects. During No-Go correct rejects, heavy drinkers relative to light drinkers showed decreased activity in the left SMA and bilateral ACC, bilateral parietal lobules, thalamus, putamen, right parahippocampal gyrus/ hippocampus, bilateral middle frontal gyrus, and left superior temporal gyrus (Figure 2a, Table 3 (primary clusters), and Supplementary Table 1 (subclusters)). Some group main-effect region of interest contrast values correlated significantly with alcohol- and impulsivity-related measures (Tables 4a and b). Maximum alcohol-consumption scores correlated negatively with regional BOLD signal changes in anterior cingulate gyrus, left postcentral, left thalamus, right middle frontal, and right putamen during No-Go correct rejects ( $v s$ implicit baseline) (Figure 2 and Table 4a). BOLD signal change in the ACC and left superior temporal gyrus correlated negatively with the number of blackouts (Figure 2 and Table 4a). Negative correlations were observed between activity in multiple main-effect ROIs (primary clusters) and impulsivity scores as noted in Table $4 \mathrm{~b}$ and Figure 2. All correlations are reported at an uncorrected $p$-value (see table footnote for details) and thereby should be interpreted with caution.

No-Go false alarms. No region survived after correction for multiple comparisons in this between-group contrast.

Within-group results showed bilateral activation patterns in both No-Go conditions and revealed largely similar patterns as observed in previously published papers from our group using the same paradigm (Kiehl et al, 2000a). fMRI patterns had substantial overlap in several regions between the two groups. Results are provided in Supplementary Figure 1.

\section{DISCUSSION}

This study evaluated neural responses to a Go/No-Go task and their relationships with alcohol- and impulsivityrelated measures in heavy- $v s$ light-alcohol-drinking college students. The major finding was that heavy drinkers had less fMRI BOLD response mainly in left SMA, ACC (Brodmann area (BA) 24), parietal lobule, thalamus, putamen, right parahippocampal gyrus, right hippocampus, right middle frontal gyrus, and left superior temporal gyrus during response inhibition. In addition, heavy drinkers had slower RTs during correct and incorrect hits compared to light drinkers, along with higher numbers of memory blackouts and higher impulsivity-related scores. Betweengroup differences in brain activations in a subset of the

Table 2 Go/No-Go Task Responses

\begin{tabular}{|c|c|c|c|c|}
\hline & Heavy drinkers $(n=35), M(S D)$ & Light drinkers $(n=56), M(S D)$ & t-test & $P$-value \\
\hline Number of correct rejections & $47.85(11.73)$ & $43.07(17.53)$ & 1.51 & NS \\
\hline Number of false alarms & $30.14(11.77)$ & $34.92(17.63)$ & 1.55 & NS \\
\hline Error proportion & $38.64 \%$ & $44.78 \%$ & 1.41 & NS \\
\hline Reaction time (ms) & $761.65(78.4 I)$ & $718.12(80.94)$ & -2.52 & 0.01 \\
\hline Go hit reaction time (ms) & 806.79 (71.73) & 753.43 (89.78) & -2.95 & 0.004 \\
\hline
\end{tabular}

Abbreviations: M, mean; NS, nonsignficant; SD, standard deviation.

Groups did not differ in numbers of correct rejections or false alarms or error proportions, but they differed in reaction times during Go correct and No-Go false alarm events. 

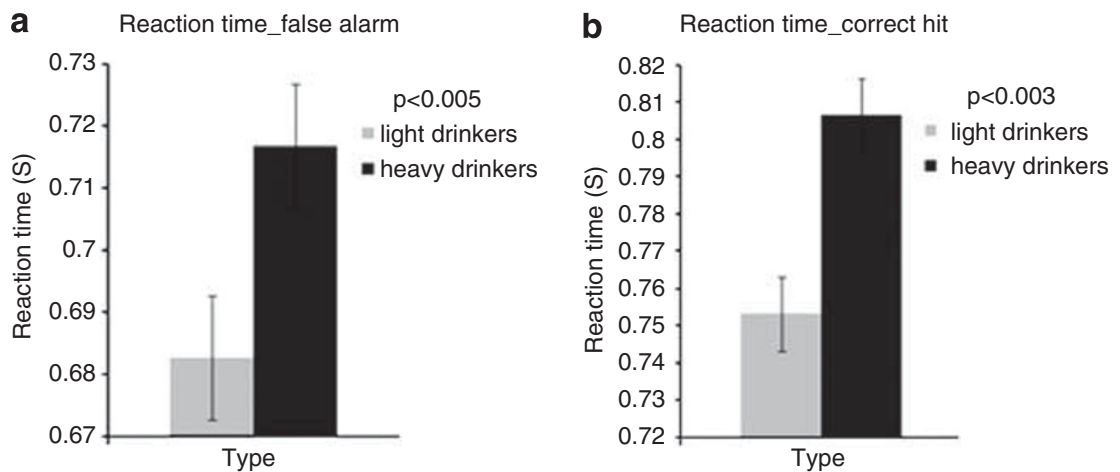

Figure I Mean (standard error) reaction time (RT) (in s) for No-Go false alarms (a) and Go correct hits (b) in the heavy and light drinker groups. The heavy drinkers exhibited significantly greater RTs for both conditions.
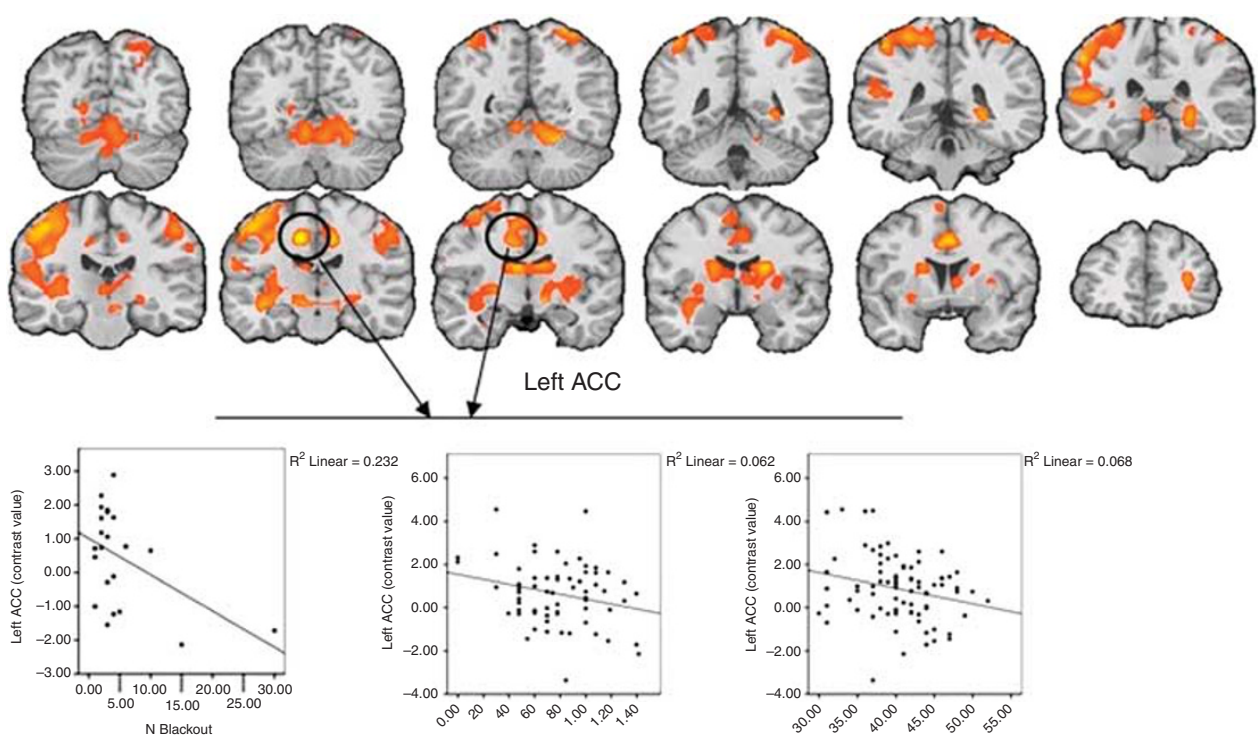

MAX-Alc_6months

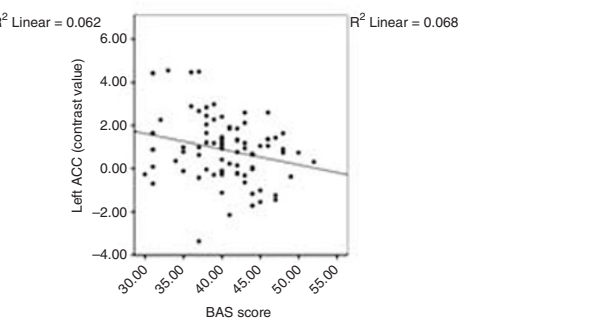

Figure 2 (Top) Significant functional magnetic resonance imaging (fMRI) between-group differences during No-Go correct rejections ( $p<0.025$, false discovery rate $(F D R) ; k=25)$. Light drinkers exhibited greater blood oxygen level-dependent (BOLD) responses than did heavy drinkers, notably in anterior cingulate gyrus (Brodmann area 24), left supplementary motor area, right parietal lobule, right hippocampus, and left superior temporal gyrus. (Below) Significant negative correlations were seen between activation in the left anterior cingulate cortex (ACC) during No-Go correct rejects and self-reported alcohol-consumption-related measures (number of blackouts, maximum number of drinks) and behavioral-activation-system scores. Individuals with heavier alcohol consumption and higher Behavioral Activation scores showed less BOLD response in right ACC than individuals with lighter consumption and lower behavioral activation.

above-reported regions correlated with alcohol- and impulsivity-related measures.

\section{Go/No-Go Behavioral Performance and fMRI Activity}

Heavy and light drinkers differed in Go correct-hit and No-Go false-alarm RTs, with heavy drinkers manifesting slower RTs in Go and No-Go conditions. This slower reaction time may represent a conflict during prepotent response inhibition resulting in response delays, consistent with previous reports (Zack et al, 2011). Nonsignificant differences in error proportion between groups further improves our interpretation of fMRI response differences seen in this study, as the data suggest that the two groups are matched on task performance at a behavioral level.

A critical interpretive issue is that heavy drinkers were slower to respond overall, in doing so they might have ironically also benefitted from committing fewer errors. To address this issue empirically, we correlated RT during error commission to proportion of errors and saw a significant negative correlation in both groups, suggesting that when subjects took more time to respond they committed fewer errors. However, importantly, when we compared the correlation coefficients between groups using a Fisher's $r-Z$ transformation method, we found no significant between-group differences, indicating that this pattern of general slowness was observed in both groups equally, thereby not causing an interpretation bias in the reported results.

Aspects of our fMRI results are consistent with our initial hypothesis of differential fMRI activity in task-relevant attention and response inhibition areas. One such example is reduced fMRI activity in left SMA and ACC, parietal lobule, right parahippocampal gyrus, right hippocampus, 
Table 3 Regions Showing Significant Effects for Heavy Drinking on BOLD Response to Correct Rejection vs Baseline (Clusters $>25$ voxels, PFDR $<0.025$ )

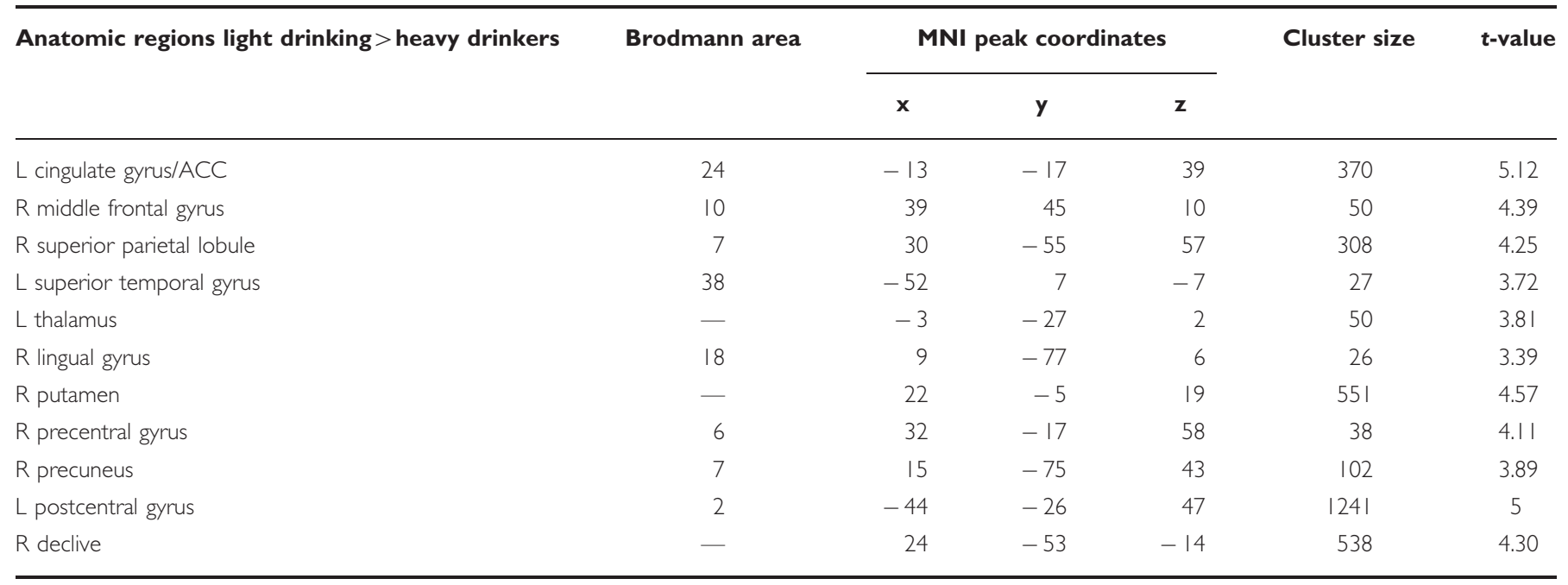

Abbreviation: $L / R$, Left/right.

The table shows post hoc $t$-test peak $t$-values in region showing main effect of group differences, the corresponding $\mathrm{MNI}$ coordinates are in mm and volumes of regions are in voxels.

right middle frontal gyrus, and left superior temporal gyrus during successful response inhibition (No-Go correct rejection $v s$ baseline) in heavy drinkers, but no significant group differences in fMRI activity for unsuccessful response inhibition (false alarms) after multiple comparisons. Our results are also compatible with previous studies of alcoholics $v s$ healthy controls that showed differential activity associated with No-Go correct rejects in the ACC (Pandey et al, 2012).

The ACC and parietal cortex are part of a distributed network that underlies cognitive control, conflict monitoring, effortful processing, and impulse control (Botvinick et al, 1999, 2001; Luu and Pederson, 2004; Ridderinkhof et al, 2004; Aron et al, 2004; Jentsch and Taylor, 1999; Lyvers, 2000; Everitt and Robbins, 2005; Bekker et al, 2005). Frontal lobe dysfunction may result from alcohol-related brain damage (Oscar-Berman and Ksenija, 2007; Dirksen et al, 2006). Anderson et al (2005) showed that greater BOLD response to inhibition during a Go/No-Go task predicted more expectancies of cognitive and motor impairments from alcohol in adolescents. These results suggest that decreased inhibitory control may contribute to more positive and less negative alcohol expectancies, which could eventually lead to problem drinking (Anderson et al, 2005). Our results regarding deficits in SMA, ACC, frontal lobe, and parietal lobule are broadly compatible with the above reports.

\section{Impulsivity-Related Measures and fMRI Activity}

Impulsivity-related constructs contribute importantly to addictions (Andrews et al, 2011; Dalley et al, 2011; Lejuez et al, 2005, 2010; Oberlin and Grahame, 2009; Petry, 2001). Individuals with familial alcoholism show increased impulsivity relative to those without (Cloninger, 1987; Ernst et al, 2006; Knop, 1985; Petry et al, 2002; Saunders et al, 2008; Sher, 1991). Thus, we hypothesized that heavy drinkers would score higher on the impulsivity-related measures used in this study. We found significant group differences on self-reported measures including the Sensation Seeking Scale (Zuckerman et al, 1979), behavioral activation system score of the BIS/BAS scale (Carver and White, 1994), and BIS-11 (Patton et al, 1995), consistent with previous results showing higher behavioral activation, impulsivity and sensation-seeking in relation to hazardous drinking, alcohol abuse/dependence, and $\mathrm{FH}$ of alcoholism (Verdejo-Garcia et al, 2008; Knop, 1985; Petry et al, 2002; Saunders et al, 2001; Sher, 1991; Cloninger, 1987; Hamilton et al, 2012; Ernst et al, 2006). Interestingly, between-group differences were not observed on the behavioral task (the BART) assessing risk-taking propensities. BART scores have previously been found to factor independently from self-reported impulsivity-related measures used in this study (Meda et al, 2009), suggesting that behavioral risktaking may be a dissociable construct from perceived assessments of impulsivity-related tendencies. As selfreported and not behavioral measures of impulsivity were found recently to mediate the relationship between stress and hazardous drinking in a community sample of adults (Hamilton et al, 2013), future studies should consider how stress exposure might interact with individual differences in impulsivity-related tendencies in the propensities of college students to consume alcohol. Given data linking both selfreported sensation-seeking and behavioral risk-taking in early adolescence (MacPherson et al, 2010) to prospective alcohol-use behaviors, the extent to which these relationships might change over time and affect college students warrants additional investigation.

Impulsivity-related measures also correlated with several regions showing differential brain activity between groups. In the No-Go correct rejection vs baseline contrast, BOLD activity in the left ACC, left thalamus, right lingual gyrus, right middle frontal, right putamen, and left postcentral gyrus correlated inversely with BAS scores. Similarly, BIS-11 and sensation-seeking scores correlated inversely with BOLD signal in the right putamen. Despite correlations 
Table 4a Correlations Between-Group Main Effect Primary ROI clusters and Alcohol Consumption Measurements

\begin{tabular}{|c|c|c|c|c|c|c|}
\hline \multirow{2}{*}{$\begin{array}{l}\text { ROls } \\
\text { L postcentral gyrus }\end{array}$} & \multirow{2}{*}{$\begin{array}{c}\begin{array}{c}\text { Brodmann } \\
\text { area }\end{array} \\
2\end{array}$} & \multicolumn{3}{|c|}{$\begin{array}{l}\text { Peak MNI } \\
\text { coordinate }\end{array}$} & \multirow{2}{*}{$\begin{array}{c}\text { Max } \\
\text { Alc } \\
-0.249^{c}\end{array}$} & \multirow{2}{*}{$\begin{array}{c}\text { Blackout } \\
\text { NS }\end{array}$} \\
\hline & & -44 & -26 & 47 & & \\
\hline$L$ thalamus & - & -3 & -27 & 2 & $-0.342^{d}$ & NS \\
\hline$L$ cingulate gyrus/ACC & 24 & -13 & -17 & 39 & $-0.250^{c}$ & $-0.482^{b}$ \\
\hline R precuneus & 7 & 15 & -75 & 43 & NS & NS \\
\hline R precentral gyrus/SMA & 6 & 32 & -17 & 58 & NS & NS \\
\hline $\begin{array}{l}\mathrm{L} \text { superior temporal } \\
\text { gyrus }\end{array}$ & 38 & -52 & 7 & -7 & NS & $-0.454^{f}$ \\
\hline R superior parietal lobule & 7 & 30 & -55 & 57 & NS & NS \\
\hline R middle frontal gyrus & 10 & 39 & 45 & 10 & $-0.293^{a}$ & NS \\
\hline R putamen & - & & -5 & 19 & $-0.363^{e}$ & NS \\
\hline $\mathrm{R}$ lingual gyrus & 18 & & -77 & 6 & NS & NS \\
\hline $\mathrm{R}$ declive & - & & -53 & -14 & NS & NS \\
\hline
\end{tabular}

Abbreviations: ACC, anterior cingulate cortex; blackout, number of alcoholrelated memory blackouts; $L / R$, left/right; Max Alc, maximum number of drinks subjects consumed in $24 \mathrm{~h}$ in past 6 months; NS, nonsignficant; ROI, regions of interest.

All $p$-values are reported at the uncorrected level.

${ }^{a} p<0.01$

${ }_{p} p<0.02$

$c_{p}<0.04$.

${ }^{d} p<0.005$

$e_{p}<0.003$

$\mathrm{f} p<0.03$. with sensation seeking and other impulsive measures, we did not find either between-group differences or correlations with the BART. This finding is consistent with the notion that the BART might index a separate impulsivityrelated construct as discussed previously (Meda et al, 2009). Despite being characterized by premorbid impulsiveness, sensation seeking, and higher rates of anxiety, it could be possible that certain internalizing features in the heavydrinking group biases their actions in a more risk-aversive manner regarding asset forfeiture and cognitive complexity, reflected by a lack of difference on tasks such as BART (Lejuez et al, 2002). Using fMRI, Claus et al (2011) investigated the neural basis of impulsive choice in AUDs, suggesting that these may result from functional anomalies in widely distributed but interconnected brain regions involved in cognitive and emotional control. Our results are compatible with previous observations pointing to dysfunction of the orbitofrontal cortex (Berlin et al, 2004; Winstanley et al, 2004), superior frontal gyrus (Horna et al, 2003), ACC, superior temporal gyrus (Garavan et al, 2002), and hippocampus (Cheung and Cardinal, 2005) as possible substrates of elevated impulsivity and behavioral activation. Acute alcohol use itself influences error processing and associated fMRI response during No-Go false alarms (Anderson et al, 2011). Animal studies of impulsive choice implicate regions including the hippocampus (Cheung and Cardinal, 2005), complementary to our findings on relationships between self-reported impulsivity and hippocampal activation during Go/No-Go performance.

Table 4b Correlations Between-Group Main Effect Primary Region-of-interest (ROI) Clusters and Impulsivity Scores

Impulsivity (Pearson's r)

\begin{tabular}{|c|c|c|c|c|c|c|c|c|c|}
\hline & & & & & \\
\hline & & & & & BAS score & BIS score & BIS-I I & Zuckerman & BART \\
\hline L ACC & 24 & -13 & -17 & 39 & $-0.24 I^{c}$ & NS & NS & NS & NS \\
\hline$L$ thalamus & & -3 & -27 & 2 & $-0.233^{c}$ & NS & $-0.362^{f}$ & NS & NS \\
\hline R lingual gyrus & 18 & 9 & -77 & 6 & $-0.268^{a}$ & NS & $-0.221^{c}$ & NS & NS \\
\hline $\mathrm{R}$ middle frontal gyrus & 10 & 39 & 45 & 10 & $-0.242^{c}$ & NS & NS & NS & NS \\
\hline L superior temporal gyrus & 38 & -52 & 7 & -7 & $-0.252^{b}$ & NS & NS & NS & NS \\
\hline R precentral gyrus/SMA & 6 & 32 & -17 & 58 & NS & NS & NS & NS & NS \\
\hline L postcentral gyrus & 2 & -44 & -26 & 47 & $-0.259^{b}$ & NS & NS & NS & NS \\
\hline $\mathrm{R}$ declive & - & 24 & -53 & -14 & $-0.413^{g}$ & NS & NS & NS & NS \\
\hline R precuneus & 7 & 15 & -75 & 43 & NS & NS & NS & NS & NS \\
\hline
\end{tabular}

Abbreviations: ACC, anterior cingulate cortex; BART, Balloon Analog Risk Task; BAS, Behavioral Activation System; BIS, Behavioral Inhibition System; BIS-I I, Barratt Impulsiveness Scale; L/R, left/right; ROI, regions of interest; Zuckerman, Sensation Seeking Scale.

All $p$-values are reported at the uncorrected level.

${ }^{a} p<0.01$.

$b_{p}<0.02$

$c_{p}<0.03$.

$\mathrm{d}_{p}<0.006$

$e_{p}<0.004$.

$f_{p}<0.001$.

$s_{p}<0.0001$. 
Taken together, our findings suggest that the neural correlates of successful response inhibition on the Go/NoGo task involve a distributed network of cortical and subcortical regions and individual differences in the degree of regional brain activation relate to out-of-magnet measures of self-reported behavioral activation, impulsivity, and sensation-seeking. Owing to the ongoing nature of this study, we do not yet have current follow-up drinking/ imaging information on our subjects, which might limit the interpretability of the results in the context of brain-behavior prediction of substance abuse. However, our study can be compared with a recently published paper of Norman et al (2011), who performed a longitudinal study using a Go-NoGo fMRI task similar to ours in an adolescent population (ages 12-14 years). Results show that at baseline (ie, before using alcohol), youth who had transitioned to heavy alcohol use had largely reduced activation during response inhibition in a core set of 12 regions that largely overlapped with the reduced regional activity reported in heavy drinkers in this study. Given that a subset of these regions also correlated with higher impulsivity scores in our subjects, it is possible that blunted activation and/or increased impulsitivity related to these regions might indicate delayed/abnormal maturation of inhibitory networks in future substance abusers. Given links between these constructs and alcohol-use patterns, the results suggest specific neural regions/circuits that might represent targets for therapeutic interventions for heavy drinking among college students.

Limitations and future directions. Our study involved several limitations. It would be useful to compare No-Go correct rejects to a Go correct-hits baseline, thereby increasing the specificity of the results to inhibition over execution. However, our design was not optimized to study activity associated with Go correct hits. Secondly, to measure BOLD correlates of response inhibition, we compared No-Go correct rejects to an implicit baseline. Although this offers some advantages over subtractive contrasts in interpretation (ie, changes in activity in subtractive contrasts can be attributed to an increase/ decrease in BOLD in one condition compared with another, or a change in both), disadvantages entail attributing results specifically to an inhibitory process. To address this, we conducted a supplementary analysis of No-Go correct rejects $>$ No-Go false alarms (successful vs unsuccessful inhibition). These results support our conclusion that our No-Go correct rejects $>$ baseline contrast indexed inhibition. However, these results are not reported here as findings were weak ( $p<0.01$ uncorrected value) and did not survive corrections for multiple comparisons. This question should be explored in future work using samples providing sufficient statistical power to address this issue conclusively. The current data also do not speak to the issue of cause $v s$ consequence; it is unclear whether different Go/No-Go behavior and brain BOLD patterns represent pre-existing vulnerabilities in response inhibition that are risk factors for later AUDs, consequences of heavy drinking, or both. To improve sample representativeness, we adopted a slightly unconventional (but previously published) method of classifying subjects into light (a mix of both AUD and non-AUD subjects) and heavy drinkers, which might have added some potential noise to the data. It is also unclear if individual differences in motivation might have confounded subjects' signal detection/perception capability. In addition, the significant differences in occurrence of depressive/ anxiety disorder rates among groups might be an additional confounding factor. Also, as fMRI-impulsivity correlations did not survive corrections for multiple comparisons, these findings need to be interpreted with caution.

As alcoholism can be associated with comorbid psychiatric symptoms, larger samples should investigate directly the impact of psychiatric comorbidity as related to the heavy-drinking-related neural correlates of response inhibition. Personality and alcohol expectancies have also been examined as potential risk factors for the initiation and maintenance of alcohol use in adolescents and young adults, and these should be examined further with respect to the heavy drinking related neural correlates of response inhibition. Nonetheless, the findings that heavy drinkers demonstrate evidence of decreased processing associated with regions subserving attention, motivation, and response inhibition during No-Go response withholding and slower $\mathrm{RT}$ s in response to an fMRI Go/No-Go task, suggest a neural mechanism that may underlie heavy drinking among college students. Future studies should consider investigating prevention and treatment strategies targeting impulsivity-related constructs, particularly as changes in impulsivity have correlated with changes in other addictive behaviors during treatment (Blanco et al, 2009).

In summary, young heavy drinkers demonstrated altered task performance, greater impulsivity-related ratings, and reduced response-inhibition-task-associated brain activity, most prominently in ACC, portions of frontal lobe, hippocampus, thalamus, and superior temporal regions, brain areas associated with impulsivity, alcoholism, and/or alcohol-related toxicity. Specific alcohol- and impulsivityrelated measures were associated with between-group differences in brain activation. Differences in impulsivityrelated tendencies, behavior, and brain activation patterns could not be attributed to group differences in alcoholism $\mathrm{FH}$ in our sample, as this measure did not differ between groups. If the observed brain activity differences in regions involved with cognitive control, attention, and response inhibition in heavy drinkers results in a reduced capacity to inhibit responses to No-Go stimuli, such individuals might require more effortful inhibitory control to overcome impulsiveness tendencies and yield equal stopping performance, and this might translate into heavier drinking in real-life situations.

\section{FUNDING AND DISCLOSURE}

Dr Pearlson has been a consultant to Bristol Myers Squibb for projects unrelated to the current research. Dr Potenza has served as a consultant or advisor to Boehringer Ingelheim, Somaxon, gambling businesses and organizations, law offices, and the federal defender's office in issues regarding impulse control disorders. He has received research support from the National Institutes of Health, Veteran's Administration, Mohegan Sun Casino, the National Center for Responsible Gaming, Psyadon, Forest Laboratories, Ortho-McNeil, Oy-Control/Biotie, and GlaxoSmithKline. He has participated in surveys, mailings, or 
telephone consultations related to drug addiction, impulse control disorders, or other topics. He has provided clinical care in the Connecticut Department of Mental Health and Addiction Services Problem Gambling Services Program. He has performed grant reviews for the National Institutes of Health and other agencies. He has guest-edited journal sections, has given academic lectures in grand rounds, continuing medical education events, and other clinical and scientific venues, and has generated book or book chapters for publishers of mental health texts. The other authors declare no conflicts of interest.

\section{ACKNOWLEDGEMENTS}

This research was supported by NIAAA grants R01AA016599 and RL1AA017539 to GP.

\section{REFERENCES}

Anderson KG, Schweinsburg A, Paulus MP, Brown SA, Tapert S (2005). Examining personality and alcohol expectancies using functional magnetic resonance imaging (fMRI) with adolescents. J Stud Alcohol 66: 323-331.

Anderson BM, Stevens MC, Meda SA, Jordan K, Calhoun VD, Pearlson GD (2011). Functional imaging of cognitive control during acute alcohol intoxication. Alcohol Clin Exp Res 35: 1-10.

Andrews MM, Meda SA, Thomas AD, Potenza MN, Krystal JH, Worhunsky P et al (2011). Individuals family history positive for alcoholism show functional magnetic resonances imaging differences in reward sensitivity that are related to impulsivity factors. Biol Psychiatry 69: 675-683.

Aron AR, Robbins TW, Poldrack RA (2004). Inhibition and the right inferior frontal cortex. Trends Cogn Sci 8: 170-177.

Barkley RA (1997). Behavioral inhibition, sustained attention, and executive functions: constructing a unifying theory of ADHD. Psychol Bull 121: 65-94.

Bauer LO (2001). CNS recovery from cocaine, cocaine and alcohol, or opioid dependence: a P300 study. Clin Neurophysiol 112: 1508-1515.

Bekker EM, Kenemans JL, Verbaten MN (2005). Source analysis of the N2 in a cued Go/NoGo task. Brain Res Cogn Brain Res 22: 221-231.

Berlin HA, Rolls ET, Kischka U (2004). Impulsivity, time perception, emotion and reinforcement sensitivity in patients with orbitofrontal cortex lesions. Brain 127: 1108-1126.

Blanco C, Potenza MN, Kim SW, Ibanez A, Zaninelli R, Saiz-Ruiz J et al (2009). A pilot study of impulsivity and compulsivity in pathological gambling. Psychiatry Res 167: 161-168.

Bornovalova M, Daughters S, Hernandez G, Richards J, Lejuez C (2005). Differences in impulsivity and risk-taking propensity between primary users of crack cocaine and primary users of heroin in a residential substance-use program. Exp Clin Psychopharmacol 13: 311-318.

Borsari J (2007). Predictors of alcohol use during the first year of college: implications for prevention. Brian Addict Behav 32: 2062-2086.

Botvinick M, Nystrom LE, Fissell K, Carter CS, Cohen JD. (1999). Conflict monitoring versus selection-for-action in anterior cingulate cortex. Nature 402: 179-181.

Botvinick MM, Braver TS, Barch DM, Carter CS, Cohen JD (2001). Conflict monitoring and cognitive control. Psychol Rev 108: 624-652.

Brandeis D, Banaschewski T, Baving L, Georgiewa P, Blanz B, Warnke A et al (2002). Multicenter P300 brain mapping of impaired attention to cues in hyperkinetic children. J Am Acad Child Adolesc Psychiatry 41: 990-998.

Cahalan D, Cisin IH, Crossley HM (1969). American drinking practices: a national study of drinking behavior and attitudes. Monogr Rutgers Center Alcohol Stud 6: 184.

Carver CS, White TL (1994). Behavioral inhibition, behavioral activation, and affective responses to impending reward and punishment: the BIS/BAS scales. J Person Soc Psychol 67: 319-333.

Chamberlain SR, Sahakian BJ (2007). The neuropsychiatry of impulsivity. Curr Opin Psychiatry 20: 255-261.

Cheung TH, Cardinal RN (2005). Hippocampal lesions facilitate instrumental learning with delayed reinforcement but induce impulsive choice in rats. BMC Neurosci 6: 36.

Claus ED, Kent AK, Kent EH (2011). Neural and behavioral mechanisms of impulsive choice in alcohol use disorder. Alcoholism Clin Exp Res 35: 1209-1219.

Claus ED, Hutchison KE (2012). Neural mechanisms of risk taking and relationships with hazardous drinking. Alcohol Clin Exp Res 36: 932-940.

Claus ED, Feldstein Ewing SW, Filbey FM, Hutchison KE (2013). Behavioral control in alcohol use disorders: relationships with severity. J Stud Alcohol Drugs 74: 141-151.

Cloninger CR (1987). Neurogenetic adaptive mechanisms in alcoholism. Science 236: 410-416.

Courtney KE, Polich J (2009). Binge drinking in young adults: data, definitions, and determinants. Psychol Bull 135: 142-156.

Courtney KE, Ghahremani DG, Ray LA (2012). Fronto-striatal functional connectivity during response inhibition in alcohol dependence. Addict Biol 18: 593-604.

Cox WM, Yeates GN, Regan CM (1999). Effects of alcohol cues on cognitive processing in heavy and light drinkers. Drug Alcohol Depend 55: 85-89.

Dager A, Anderson BM, Stevens MC, Pulido C, Rosen R, JiantonioKelly RE et al (2013). Influence of alcohol use and family history of alcoholism on neural response to alcohol cues in college drinkers. Alcohol Clin Exp Res 37(Suppl 1): E161-E171.

Dalley JW, Everitt BJ, Robbins TW (2011). Impulsivity, compulsivity and top-down control. Neuron 69: 680-694.

Dirksen CL, Howard JA, Cronin-Golomb A, Oscar-Berman M (2006). Patterns of prefrontal dysfunction in alcoholics with or without Korsakoff's syndrome, patients with Parkinson's disease, and patients with rupture and repair of the anterior communicating artery. Neuropsychiatr Dis Treat 23: 327-339.

Ernst M, Luckenbaugh DA, Moolchan ET, Leff MK, Allen R, Eschel $\mathrm{N}$ et al (2006). Behavioral predictors of substance-use initiation in adolescents with and without attention-deficit/hyperactivity disorder. Pediatrics 117: 2030-2039.

Everitt BJ, Robbins TW (2005). Neural systems of reinforcement for drug addition: from actions to habits to compulsion. Nat Neurosci 8: 1481-1489.

Fillmore MT, Rush CR (2002). Impaired inhibitory control of behavior in chronic cocaine users. Drug Alcohol Depend 66: 265-273.

First MB, Spitzer RL, Gibbon M, Williams JBW (2002). Structured Clinical Interview for DSM-IV-TR Axis I Disorders-Research Version, Non-Patient Edition (SCID-I/NP, 11/2002 Revision). Biometrics Research Department, New York State Psychiatric Institute: New York, NY, USA.

Frank Y, Seiden JA, Napolitano B (1998). Electrophysiological changes in children with learning and attentional abnormalities as a function of age: event-related potentials to an 'oddball' paradigm. Clin Electroencephalogr 29: 188-193.

Freire L, Roche A, Mangin JF (2002). What is the best similarity measure for motion correction in fMRI time series? IEEE Trans Med Imag 21: 470-484.

Garavan H, Ross TJ, Murphy K, Roche RA, Stein EA (2002). Dissociable executive functions in the dynamic control of 
behavior: inhibition, error detection, and correction. NeuroImage 17: 1820-1829.

Goldstein RZ, Volkow ND (2002). Drug addiction and its underlying neurobiological basis: neuroimaging evidence for the involvement of the frontal cortex. Am J Psychiatry 159: 1642-1652. Goodwin DW (1995). Alcohol amnesia. Addiction 90: 315-317.

Hamilton KR, Ansell EB, Reynolds B, Potenza MN, Sinha R (2013). Self-reported impulsivity, but not behavioral choice or response impulsivity, partially mediates the effect of stress on drinking behavior. Stress 16: 3-15.

Hamilton KR, Sinha R, Potenza MN (2012). Hazardous drinking and dimensions of impulsivity, behavioral approach, and inhibition in adult men and women. Alchol Clin Exp Res 36: 434-449.

Horna NR, Dolan M, Elliott R, Deakin JFW, Woodruff PWR (2003). Response inhibition and impulsivity: an fMRI study. Neuropsychologia 41: 1959-1966.

Jentsch JD, Taylor JR (1999). Impulsivity resulting from frontostriatal dysfunction in drug abuse: implications for the control of behavior by reward-related stimuli. Psychopharmacology 146: 373-390.

Josephs O, Turner R, Friston KJ (1997). Event related fMRI. Hum Brain Mapp 5: 243-248.

Kahler CW, Strong DR, Read JP (2005). Toward efficient and comprehensive measurement of the alcohol problems continuum in college students: the brief young adult alcohol consequences questionnaire. Alcohol Clin Exp Res 29: 1180-1189.

Kaiser S, Unger J, Kiefer M, Markela J, Mundt C, Weisbrod M (2003). Executive control deficit in depression: event-related potentials in a Go/Nogo task. Psychiatry Res 122: 169-184.

Kaufman JN, Ross TJ, Stein EA, Garavan H (2003). Cingulate hypoactivity in cocaine users during a GO NOGO task as revealed by event-related functional magnetic resonance imaging. J Neurosci 23: 7839-7843.

Kiehl KA, Smith AM, Hare RD, Liddle PF (2000a). An event-related potential investigation of response inhibition in schizophrenia and psychopathy. Biol Psychiatry 48: 210-221.

Kiehl KA, Liddle PF, Hopfinger JB (2000b). Error processing and the rostral anterior cingulate: an event-related fMRI study. Psychophysiology 37: 216-223.

Kouri EM, Lukas SE, Mendelson JH (1996). P300 assessment of opiate and cocaine users: effects of detoxification and buprenorphine treatment. Biol Psychiatry 40: 617-628.

Knop J (1985). Premorbid assessment of young men at high risk for alcoholism. Recent Dev Alcohol 3: 53-64.

Kreek MJ, Nielsen DA, Butelman ER, LaForge KS (2005). Genetic influences on impulsivity, risk taking, stress responsivity and vulnerability to drug abuse and addiction. Nat Neurosci 8: 1450-1457.

Lansbergen MM, Böcker KBE, Bekker EM, Kenemans JL (2007a). Neural correlates of stopping and self-reported impulsivity. Clin Neurophysiol 118: 2089-2103.

Lansbergen MM, Schutter DJ, Kenemans JL (2007b). Subjective impulsivity and baseline EEG in relation to stopping performance. Brain Res 11: 48-161.

Lejuez CW, Read JP, Kahler CW, Richards JB, Ramsey SE, Stuart GL et al (2002). Evaluation of a behavioral measure of risk-taking: the Balloon Analogue Risk Task (BART). J Exp Psychol 8: 75-84.

Lejuez C, Aklin W, Bornovalova M, Moolchan E (2005). Differences in risk-taking propensity across inner-city adolescent ever- and never-smokers. Nicotine Tob Res 7: 71-79.

Lejuez CW, Magidson JF, Mitchell SH, Sinha R, Stevens MC, de Wit H (2010). Behavioral and biological indicators of impulsivity in the development of alcohol use, problems and disorders. Alcohol Clin Exp Res 34: 1334-1345.

Luu P, Pederson S (2004). The anterior cingulate cortex: actions in context. In: Posner MI (ed) Cognitive Neuroscience of Attention. Guilford: New York, NY, USA, pp 232-242.
Lyvers M. (2000). 'Loss of control' in alcoholism and drug addiction: a neuroscientific interpretation. Exp Clin Psychopharmacol 8: 225-249.

MacPherson L, Magidson JF, Reynolds EK, Kahler CW, Lejuez CW (2010). Changes in sensation seeking and risk-taking propensity predict increases in alcohol use among early adolescents. Alcohol Clin Exp Res 34: 1400-1408.

Marczinski CA, Combs SW, Fillmore MT (2007). Increased sensitivity to the disinhibiting effects of alcohol in binge drinkers. Psychol Addict Behav 21: 346-354.

Meda SA, Stevens MC, Potenza MD, Pittman B, Gueorguieva R, Andrews MM et al (2009). Investigating the behavioral constructs of impulsivity domains using principal component analysis. Behav Pharmacol 20: 390-399.

Norman AL, Pulido C, Squeglia LM, Spadoni AD, Paulus MP, Tapert SF (2011). Neural activation during inhibition predicts initiation of substance use in adolescence. Drug Alcohol Depend 119: $216-223$.

Oberlin BG, Grahame NJ (2009). High-alcohol preferring mice are more impulsive than low-alcohol preferring mice as measured in the delay discounting task. Alcohol Clin Exp Res 33: 1294-1303.

Oscar-Berman M, Ksenija M (2007). Alcohol: effects on neurobehavioral functions and the brain. Neuropsychol Rev 17: 239-257.

Pandey AK, Kamarajan C, Tang Y, Chorlian DB, Roopesh BN, Manz N et al (2012). Neurocognitive deficits in male alcoholics. An ERP/sLORETA analysis of the N2 component in an equal probability Go/No-Go task). Biol Psychol 89: 170-182.

Patton JM, Stanford MS, Barratt ES (1995). Factor structure of the Barratt Impulsiveness Scale. J Clin Psychol 51: 768-774.

Perry PJ, Argo TR, Barnett MJ, Liesveld JL, Liskow B, Hernan JM et al (2006). The association of alcohol-induced blackouts and grayouts to blood alcohol concentrations. J Forens Sci 51: 896-899.

Petry NM (2001). Delay discounting of money and alcohol in actively using alcoholics, currently abstinent alcoholics, and controls. Psychopharmacology 154: 243-250.

Petry NM, Kirby KN, Kranzler HR (2002). Effects of gender and family history of alcohol dependence on a behavioral task of impulsivity in healthy subjects. J Stud Alcohol 63: 83-90.

Pliszka SR, Liotti M, Woldorff MG (2000). Inhibitory control in children with attention-deficit/hyperactivity disorder: eventrelated potentials identify the processing component and timing of an impaired right-frontal response-inhibition mechanism. Biol Psychiatry 48: 238-246.

Posner MI, DiGirolamo G (1998). Executive attention: conflict, target detection and cognitive control. In: Parasuraman $\mathrm{R}$ (ed.) The Attentive Brain. MIT Press: Cambridge, MA, USA, pp 401-423.

Potenza MN, De Wit H (2010). Control yourself: alcohol and impulsivity. ACER 34: 1303-1305.

Ridderinkhof KR, Ullsperger M, Crone EA, Nieuwenhuis S (2004). The role of the medial frontal cortex in cognitive control. Science 306: 443-447.

Rubia K, Oosterlaan J, Sergeant JA, Brandeis D, van Leeuwen T (1998). Inhibitory dysfunction in hyperactive boys. Behav Brain Res 94: 25-32.

Rubia K, Russell T, Overmeyer S, Brammer MJ, Bullmore ET, Sharma $\mathrm{T}$ et al (2001). Mapping motor inhibition: conjunctive brain activations across different versions of go/no-go and stop tasks. NeuroImage 13: 250-261.

Saccone NL, Kwon JM, Corbett J, Goate A, Rochberg N, Edenberg HJ et al (2000). A genome screen of maximum number of drinks as an alcoholism phenotype. Am J Med Genet 96: 632-637.

SAMHSA (2011). Results from the (2010) National Survey on Drug Use and Health: Summary of National Findings, in Series Results from the 2010 National Survey on Drug Use and Health: Summary of National Findings, NHSDA Series H-41, Substance Abuse and Mental Health Services Administration. SAMHSA: Rockville, MD, USA. 
Saunders B, Schneider F, Habel U, Wagner M, Franke P, Salloum JB et al (2001). Subcortical correlates of craving in recently abstinent alcoholic patients. Am J Psychiatry 158: 1075-1083.

Saunders B, Farag N, Vincent AS, Collins FL Jr, Sorocco KH, Lovallo WR (2008). Impulsive errors on a Go-NoGo reaction time task: disinhibitory traits in relation to a family history of alcoholism. Alcohol Clin Exp Res 32: 888-894.

Schweinsburg AD, McQueeny T, Nagel BJ, Eyler LT, Tapert SF (2010). A preliminary study of functional magnetic resonance imaging response during verbal encoding among adolescent binge drinkers. Alcohol 44: 111-117.

Sheehan DV, Lecrubier Y, Sheehan KH, Amorim P, Janavs J, Weiller E et al (1998). The Mini-International Neuropsychiatric Interview (M.I.N.I.): the development and validation of a structured diagnostic psychiatric interview for DSM-IV and ICD-10. J Clin Psychiatry 59(Suppl 20): 22-33.

Sher KJ (1991). Psychological characteristics of children of alcoholics. Overview of research methods and findings. Recent Dev Alcohol 9: 301-326.

Slutske WS, True WR, Scherrer JF, Heath AC, Bucholz KK, Eisen SA et al (1999). The heritability of alcoholism symptoms: 'indicators of genetic and environmental influence in alcohol-dependent individuals' revisited. Alcohol Clin Exp Res 23: 759-769.

Squeglia LM, Spadoni AD, Infante MA, Myers MG, Tapert SF (2009). Initiating moderate to heavy alcohol use predicts changes in neuropsychological functioning for adolescent girls and boys. Psychol Addict Behav 23: 715-722.

Smith EE, Jonides J (1999). Storage and executive processes in the frontal lobes. Science 283: 1657-1661.

Spear LP (2000). The adolescent brain and age-related behavioral manifestations. Neurosci Biobehav Rev 24: 417-463.

Stevens MC, Kiehl KA, Pearlson GD, Calhoun VD (2007). Functional neural networks underlying response inhibition in adolescents and adults. Behav Brain Res 181: 12-22.
Stevens MC, Kiehl KA, Pearlson GD, Calhoun VD (2009). Brain network dynamics during error commission. Hum Brain Mapp 30: 24-37.

Tomlinson KL, Brown SA, Abrantes A (2004). Psychiatric comorbidity and substance use treatment outcomes of adolescents. Psychol Addict Behav 18: 160-169.

Verdejo-Garcia A, Lawrence AJ, Clark L (2008). Impulsivity as a vulnerability marker for substance-use disorders: review of findings from high-risk research, problem gamblers and genetic association studies. Neurosci Biobehav Rev 32: 777-810.

Volkow ND, Hitzemann R, Wang GJ, Fowler JS, Wolf AP, Dewey SL (1992). Long-term frontal brain metabolic changes in cocaine abusers. Synapse 11: 184-190.

Wallsten TS, Pleskac TJ, Lejuez CW (2005). Modeling behavior in a clinically diagnostic sequential risk-taking task. Psychol Rev 112: 862-880.

Wechsler H, Molnar BE, Davenport AE, Baer JS (1999). College alcohol use: a full or empty glass? J Am Coll Health 47: 247-252.

Weisbrod M, Kiefer M, Marzinzik F, Spitzer M (2000). Executive control is disturbed in schizophrenia: evidence from eventrelated potentials in a Go/NoGo task. Biol Psychiatry 47: 51-60.

Wetherill RR, Castro N, Squeglia LM, Tapert SF (2013). Atypical neural activity during inhibitory processing in substance-naive youth who later experience alcohol-induced blackouts. Drug Alcohol Depend 128: 243-249.

Winstanley CA, Theobald DE, Cardinal RN, Robbins TW (2004). Contrasting roles for basolateral amygdala and orbitofrontal cortex in impulsive choice. J Neurosci 24: 4718-4722.

Zack M, Woodford TM, Tremblay AM, Steinberg L, Zawertailo LA, Busto UE (2011). Stress and alcohol cues exert conjoint effects on Go and stop signal responding in male problem drinkers. Neuropsychopharmocology 36: 445-458.

Zuckerman M, Eysenck SBJ, Eysenck HJ (1979). Sensation seeking in England and America: cross-cultural, age, and sex comparisons. J Consult Clin Psychol 46: 139-149.

Supplementary Information accompanies the paper on the Neuropsychopharmacology website (http://www.nature.com/npp) 